\title{
The effects of fixed-ratio sample requirements on matching to sample in the pigeon*
}

\author{
ROBERT A. SACKS, ALAN C. KAMIL, and RICHARD MACK \\ University of Massachusetts, Amherst, Mass. 01002
}

Eight Carneaux pigeons were trained on a zero-delay matching-to-sample task in which $1,10,20$, or 40 responses were required to the sample before presentation of the comparison stimuli. The results indicated increased speed of acquisition as a direct function of the FR sample requirement. In addition, increasing the number of required sample responses facilitated performance in both delayed matching and in a transfer task in which novel stimuli were introduced. These results are discussed in terms of the strengthening of mediating events associated with each sample.

Mack (1971) recently tested a group of four pigeons in a zero-delay matching-to-sample task in which each $S$ was required to peck the sample stimulus 16 times on each trial (FR 16 sample requirement) before the comparison stimuli were presented. The results indicated that these birds acquired the matching task much more rapidly than had previously been observed in experiments in which only one response was required to the sample (Sacks, 1970; Cumming, Berryman, \& Cohen, 1965). Mack's results also indicated better matching performance following the introduction of a delay interval between sample offset and onset of the comparison stimuli, as well as more rapid transfer to another matching problem using two novel hues as stimuli, when compared to FR 1 results. However, Mack had tested this group as a control for other experimental conditions in which the number of pecks required to the sample was different for the different sample stimuli. It was, therefore, impossible to evaluate the performance of the FR 16 sample requirement birds directly with the performance of FR 1 Ss. The present experiment was undertaken to investigate the effects of varying the number of responses required to the sample during the acquisition of matching to sample, the introduction of delayed matching, and transfer to new stimuli.

\section{SUBJECTS}

The Ss were eight male White Carneaux pigeons, 8-12 months of age, obtained from the Palmetto Pigeon Plant. They were maintained at $75 \pm 3 \%$ of their free feeding weight

\footnotetext{
* Supported in part by a grant from the Graduate Research Council, University of Massachusetts, Amherst, and in part by Training Grant MH-11823 from the National Institutes of Health. We wish to thank Wendy Danto for her able assistance in collecting the data.
}

for 7 days prior to and throughout the experiment by controlled daily feedings of Purina Pigeon Grains.

\section{APPARATUS}

The operant chamber was a Lehigh Valley Electronics (Model 132-02) cubicle. Three transparent Lehigh Valley keys (121-15) were mounted on one wall, $8 \mathrm{~cm}$ apart and $25 \mathrm{~cm}$ above the floor. An IEE multiple stimulus projector (with Pattern No. 696, Type 1820 bulbs) was mounted directly behind each key. A food magazine, mounted $6.5 \mathrm{~cm}$ below the center key, was illuminated whenever food (Purina Pigeon Grains) was available. A houselight was mounted on top of the intelligence panel in a manner which ensured that it would not shine directly on the keys. White noise from a speaker mounted inside the chamber was always present. All stimulus events and contingencies, as well as data recording, were arranged by a Lehigh Valley Electronics INTERACT computer system located in another room. All time intervals were reproduced to an accuracy of $10 \mathrm{msec}$.

\section{PROCEDURE}

After 2-3 days of habituation to the operant chamber, each pigeon was magazine trained by presentations of $5 \mathrm{sec}$ access to grain. The average interval between presentations was $1 \mathrm{~min}$. During both habituation and magazine training, all keys were dark and the houselight was on. During pretraining, the pecking response was shaped by approximation to one of the side keys. Half the birds were initially trained to peck the right key and half the left. Each peck of a lit key was reinforced with $3 \mathrm{sec}$ access to grain. Each pigeon was allowed 16 reinforced responses on each side key. Only one key was illuminated at any time, in random sequence, with the provision that each key was lighted 16 times. Throughout this phase, only white light was used. The center key was then illuminated with white light, and the pigeon was allowed to receive
16 reinforcements with the number of responses required for each reinforcement equal to the $F R$ sample requirement to be used with that $S$ during matching. On the following day, each $S$ again received 16 reinforcements on each key in the same fashion as on the preceding day. During this second pretraining session, the reinforcement was $2 \mathrm{sec}$ access to grain.

Following pretraining, matching-to-sample training was begun with red and green stimuli. Each trial began with the onsetof a red ( $R$ ) or green (G) sample stimulus on the center key. The appropriate number of responses to this key resulted in the removal of the sample and the presentation of the comparison stimuli on the side keys. Two pigeons were tested at each of four FR sample requirements: FR 1, FR 10, FR 20, and FR 40. A response to the correct (matching) comparison stimulus was reinforced with $2 \mathrm{sec}$ access to grain. An incorrect response produced a 10-sec blackout during which all light in the chamber were extinguished. A new trial began immediately after either food presentation or blackout. There were four possible stimulus configurations which could appear on any given trial: $R^{*} R G, G R R^{*}, G^{*} G R$, and $\mathrm{RGG}^{*}$ (where the asterisk represents the reinforced comparison stimulus). Each configuration was presented 28 times in random sequence during each daily 112-trial session. All birds were exposed to this procedure until a criterion of $85 \%$ correct matching or better was maintained for three consecutive sessions.

Delay testing began in the session following the completion of acquisition training. The procedure during this phase was identical to that of acquisition except that a delay interval, during which all keys were dark but the houselight remained on, was introduced between the offset of the sample and the onset of the comparison stimuli. Delays of $0.5,1$, 2,4 , and $8 \mathrm{sec}$ were tested. Each $\mathrm{S}$ received one session at each delay interval, in ascending order, until the series was completed or until performance approached $50 \%$ at a delay interval of 2 sec or longer. Any session in which the percentage of matching responses was below $85 \%$ was followed by zero-delay sessions until matching performance again equaled or exceeded $85 \%$.

Following the completion of delay testing, all pigeons were tested for transfer. The procedure during the transfer phase was identical to that of acquisition except that blue and yellow were substituted for red and green as stimuli. 


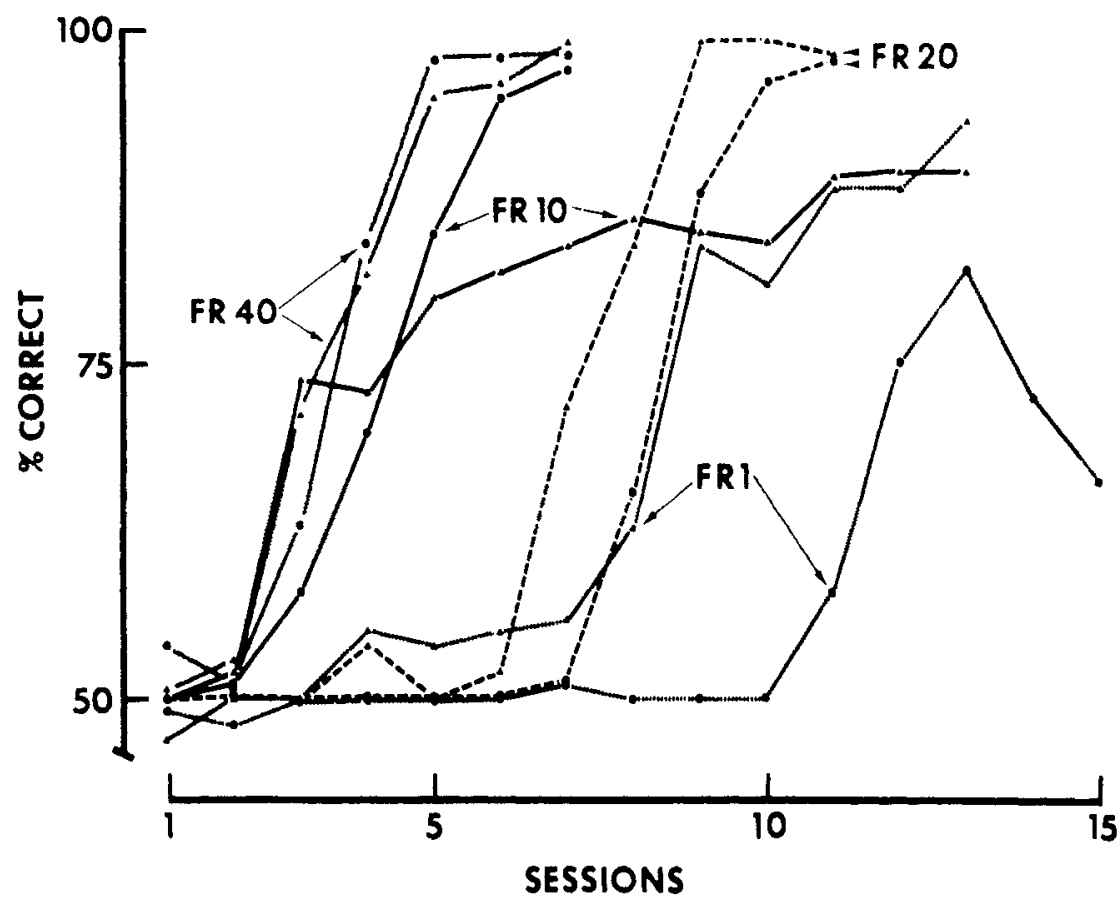

Fig. 1. Percentage correct matching during each session of acquisition for each pigeon. One FR 1 bird required 27 sessions, but only the first 15 are shown.

\section{RESULTS}

The results for each bird during acquisition. are shown in Fig. 1. Although there is some variability in these data, especially for the FR 1 and FR 10 sample requirement pigeons, these results suggest that increasing the sample response requirement increased speed of acquisition. Both FR 40 birds required 6 sessions to complete acquisition, while the FR 1 birds required 13 and 27 sessions. Previous results in this laboratory with an FR 1 sample requirement have varied considerably among pigeons, but acquisition to criterion has never taken less than 12 sessions.

The results for each bird during delay testing are shown in Fig. 2. It is clear that matching performance after delays improved with increasing FR sample requirements. While FR 1 birds were performing at chance levels after a 2 -sec delay, both FR 40 birds were above $90 \%$ correct after a 2 -sec delay and above $75 \%$ correct after an 8 -sec delay.

The results of the transfer test, shown in Fig. 3, indicate a clear facilitation of performance with higher FR sample requirements. The major effect, in terms of trials or sessions to criterion, is between the FR 1 requirement and the higher FR requirements. Perhaps more importantly, only pigeons exposed to sample requirements of FR 10 or above demonstrated above-chance matching during the first transfer session. During Sessions 1 and 2 of transfer, the relationship between percentage correct matching and size of the FR sample requirement was a monotonic increasing function of the size of the FR requirement. One FR 40 bird performed at $85 \%$ correct in Session 1 and completed transfer testing in the minimum number of sessions possible-three. It should be noted that above-chance performance during Session 1 of transfer has never been observed with an FR 1 sample requirement in this laboratory.

\section{DISCUSSION}

It seems clear that increasing an FR sample requirement increased the speed of acquisition and transfer in matching to sample, as well as improving performance when delayed matching was introduced. Berryman, Cumming, \& Nevin (1963) required an FR 5 on the sample and found no particular increase in performance compared to FR 1 conditions. There seem to be two possible explanations for this difference in result. First, FR 5 may simply be too small a sample requirement to detect the effects of increasing FR requirements. Second, Berryman et al (1963) employed a simultaneous matching procedure in which the sample remained on while the choice response was made. Thus, it is quite possible that different $F R$ sample requirements affect performance in zero-delay matching but not in simultaneous matching. A second experiment (Cumming, Berryman, \& Cohen, 1965) also failed to find any facilitative effect of increasing the FR sample requirement, in this case in zero-delay matching. However, Cumming et al (1965) did not introduce the FR requirement until the 13 th session of transfer testing. As Cumming et al pointed out, this extensive previous training with FR 1 on the sample may have obscured any positive effects of the increased FR sample requirement because such a change may have disrupted an already well-established chain of "peck center once then peck side."

The possible mechanism through which the effects of increased FR sample requirements operate must be considered. We have elsewhere (Kamil $\&$ Sacks, in press) suggested that the coding hypothesis (Cumming et al, 1965 ) offers the most satisfactory context in which to view zero-delay matching phenomena. According to this hypothesis, the choice response on individual zero-delay matching trials is under the control of specific mediating stimuli associated with each sample. This type of hypothesis seems necessary since the sample is not present when the choice response is made and therefore cannot directly and immediately control choice responding. In these terms, the effects of the increased FR sample requirements can be thought of as due to increasing the strength of the mediating events controlling the choice responses made to the comparison stimuli. The delay data particularly support this position since they seem to clearly indicate that the stimuli controlling choice responding in trained pigeons are effective longer

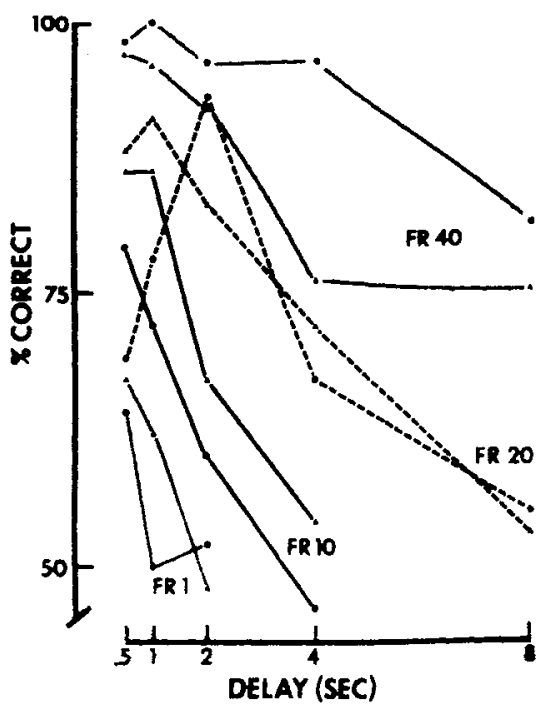

Fig. 2. Percentage correct matching at each delay interval tested for each pigeon. 


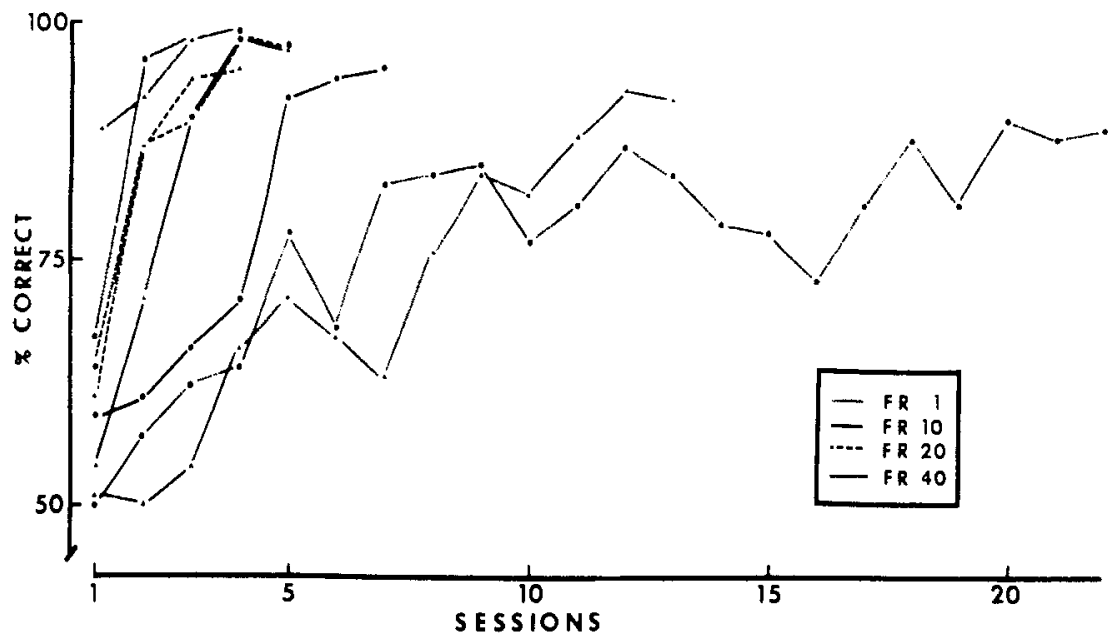

Fig. 3. Percentage correct matching for each pigeon during each session of transfer testing.

with the higher FR sample requirements. However, this statement still leaves many unresolved questions. Is the FR sample effect primarily due to increased time of exposure to the sample, to increased effort, or to some sort of an increase in the probability of attending to the critical dimension of the stimuli (color in this experiment)? Clearly many possibilities remain which must be subjected to empirical test. ${ }^{1}$

The results of the present experiment are quite similar to those obtained by Williams (1971a) in nonspatial delayed alternation. In this experiment, the color not responded to on the previous trial was correct, regardless of its position. Williams found that increasing $F R$ requirements during alternation acquisition and transfer greatly facilitated performance of his pigeons as compared to an FR 1 condition. The nonspatial delayed alternation task is similar to the zero-delay matching task in that the external stimulus upon which the choice response can be based is not present when that response is made. Williams suggested that this effect in delayed alternation might be due to the suppression of error tendencies during the performance of the fixed ratio to the choice stimuli. However, in matching to sample this cannot be a factor since $F R$ sample requirements are performed to the only stimulus available and do not constitute a choice response. Thus FR sample requirements offer no opportunity for the suppression of error tendencies. Therefore, if the effects of FR requirements in both matching and delayed alternation can be understood in terms of the same mechanism, then Williams' explanation seems unlikely. In addition, the present results clearly contradict Williams' (1971b) conclusion that "it is during the choice between stimuli that the FR variable is important [p. 19]." Clearly, the present experiment has demonstrated a large effect of the FR variable before the choice interval of the trial. Of course, the effects of FR requirements during the choice interval of each matching-to-sample trial (an FR comparison requirement) remain an open question at present.

\section{REFERENCES}

BERRYMAN, R. CUMMING，W，W， \& NEVIN, J. A. Acquisition of delayed matching in the pigeon. Journal of the Experimental Analysis of Behavior, 1963, 6. 101-107.

CUMMING, W, W. BERRYMAN, R., \& COHEN, L. R. Acquisition and transfer of zero-delay matching. Psychological Reports, 1965, 17, 435-445.

KAMIL, A. C., \& SACKS, R. A. Three-configuration matching-to-sample in the pigeon. Journal of the Experimental Analysis of Behavior, in press.

MACK, R. P. An inquiry of mediating events in the solution of the matching-to-sample problem. Unpublished Master's thesis, University of Massachusetts, 1971.

SACKS, R. A. The coding hypothesis and zero delay matching-to-sample in the pigeon. Unpublished Master's thesis, University of Massachusetts, 1971.

WIL LI AMS, B. A. Color alternation learning in the pigeon under fixed-ratio schedules of reinforcement. Joumal of the Experimental Analysis of Behavior, 1971a, 15, 129-140.

WILLIAMS, B. A. Non-spatial delayed alternation by the pigeon. Joumal of the Experimental Analysis of Behavior, 1971b, 16, 15-21.

$$
\text { NOTE }
$$

1. One possible peripheral mediator was pointed out to us by a reviewer: successive color contrast. The high $F R$ requirements increased exposure time to the colored sample stimulus. Such extended exposure could have resulted in an afterimage persisting for several seconds. If this occurred, the pigeon could have made his choice response on the basis of an apparent difference in the saturation of the comparison stimuli. This seems unlikely, since the stimuli were not very bright and the presence of the houselight would tend to diminish any afterimage. However, the operation of such peripheral mediators may provide exactly the type of mechanism specified by the coding hypothesis. 